\title{
Relevanz und Auswirkungen der 3. BtMVVÄndV für die Opioidsubstitutionstherapie
}

Ergebnisse einer qualitativen Befragung von substituierenden Ärztinnen und Ärzten

\section{Relevance and Effects of the $3^{\text {rd }}$ Revision of the Narcotic Drugs Prescription Ordinance on routine OST care}

\author{
Results of a Qualitative Survey of Substituting Physicians
}

\section{(ㄷ) (i) (ㄹ) $\ominus$}

Autoren

Kirsten Lehmann, Silke Kuhn, Bernd Schulte, Uwe Verthein

Institut

Universitätsklinikum Hamburg-Eppendorf, Zentrum für Interdisziplinäre Suchtforschung der Universität Hamburg (ZIS)

Schlüsselwörter

BtMVV, BtMVVÄndV, Evaluation, Opioidabhängigkeit, Opioidsubstitutionstherapie

Key words

Narcotic Drugs Prescription Ordinance, evaluation, opioid dependence, opioid substitution therapy, OST

online publiziert $\quad 24.2 .2021$

Bibliografie

Suchttherapie 2022; 23: 33-43

DOI 10.1055/a-1330-6349

ISSN 1439-9903

(c) 2021. The Author(s).

This is an open access article published by Thieme under the terms of the Creative Commons Attribution-NonDerivative-NonCommercial-License, permitting copying and reproduction so long as the original work is given appropriate credit. Contents may not be used for commecial purposes, or adapted, remixed, transformed or built upon. (https://creativecommons. org/licenses/by-nc-nd/4.0/)

Georg Thieme Verlag KG, Rüdigerstraße 14,

70469 Stuttgart, Germany

Korrespondenzadresse

Kirsten Lehmann

Universitätsklinikum Hamburg-Eppendorf

Zentrum für Interdisziplinäre Suchtforschung der

Universität Hamburg (ZIS)

Klinik für Psychiatrie und Psychotherapie

Martinistraße 52

20246 Hamburg

Deutschland

k.lehmann@uke.de

\section{ZUSAMMENFASSUNG}

Hintergrund Die Opioidsubstitutionstherapie (OST) ist in Deutschland die wichtigste Therapieoption zur Behandlung der Opioidabhängigkeit. Trotz ihrer Effektivität limitieren strukturelle Rahmenbedingungen und die Zurückhaltung v. a. jüngerer ÄrztInnen, die Substitution anzubieten oder fortzuführen, die Ausweitung dieser Behandlung. Mit der 3. Verordnung zur Änderung der Betäubungsmittel-Verschreibungsverordnung (3. BtMVVÄndV) wurden 2017 umfassende Reformen der Rahmenbedingungen der Opioidsubstitutionstherapie verabschiedet. In dem vorliegenden Artikel werden die Ergebnisse leitfadengestützter Interviews mit substituierenden ÄrztInnen als ein Bestandteil einer umfassenden Evaluation dieser Änderungen vorgestellt.

Methode Im Zeitraum zwischen Dezember 2019 bis April 2020 wurden in den deutschen Bundesländern Hamburg, Nordrhein-Westfalen, Sachsen und Bayern insgesamt 31 qualitative Interviews mit substituierenden ÄrztInnen geführt. Der Interviewleitfaden beinhaltete Fragen zu den in der 3. BtMVVÄndV beschlossenen Neuerungen, zu strukturellen Voraussetzungen sowie Vorstellungen für eine zukünftige Gestaltung der Opioidsubstitutionstherapie.

Ergebnisse Das Durchschnittsalter der interviewten Ärztlnnen lag bei 58,5 Jahren, $77,4 \%$ waren männlich und $54,8 \%$ konsiliarisch tätig. Die Veränderungen der Substitutionsrichtlinie werden überwiegend positiv bewertet; bezogen auf die praktische Relevanz bestehen jedoch bundeslandbezogene Unterschiede. Eine hohe praktische Relevanz wird der Ausdehnung der Take-Home-Verordnung auf 30 Tage, der Ausnahme vom Sichtbezug auf 5 Tage sowie dem veränderten Umgang mit Beikonsum zugeschrieben. Von untergeordneter Bedeutung ist die Möglichkeit, nunmehr 10 anstatt 3 PatientInnen unter der Konsiliarregelung behandeln zu dürfen.

Schlussfolgerungen Die veränderten Vorschriften dürften nicht ausreichen, um neue ÄrztInnen für die Opioidsubstitutionstherapie zu gewinnen. Kooperationen mit Apotheken, Pflegeeinrichtungen und staatlichen Institutionen werden zu- 
künftig an Bedeutung gewinnen. Ferner ist eine Unterstützung niedergelassener ÄrztInnen durch Substitutionsambulanzen, Psychiatrische Institutsambulanzen sowie Bezirkskrankenhäuser erforderlich. Der Behandlung von Abhängigkeitserkrankungen sollte in der Ausbildung von MedizinerInnen ein größerer Stellenwert zukommen.

\section{ABSTRACT}

Background Opioid substitution treatment (OST) is the most important treatment option for opioid dependence in Germany. Despite its effectiveness, structural framework conditions, as well as the reluctance of in particular younger physicians to offer or continue opioid substitution treatment, limits the expansion of this treatment. In 2017, comprehensive reforms for opioid substitution treatment were adopted with the $3^{\text {rd }}$ revision of the Narcotic Drugs Prescription Ordinance coming into force. As part of an evaluation study of these changes, this article presents the results of guideline-based interviews with substituting physicians.

Methods Between December 2019 and April 2020, a total of 31 qualitative interviews with substituting physicians were conducted in German states of Hamburg, North Rhine-Westphalia, Saxony and Bavaria. The interview guidelines contained questions on the innovations adopted in the 3rd revision of the Narcotic Drugs Prescription Ordinance, on structural preconditions as well as ideas for the future organization of opioid substitution treatment.

Results The average age of the interviewed physicians was 58.5 years, $77.4 \%$ were male and $54.8 \%$ worked as liaison and consultant. The changes due to the new substitution guideline are assessed positively. However, there are differences between the federal states in terms of practical relevance. The regulation that extends take-home opioid substitution medication to a maximum of 30 days, the exception from the direct consumption of opioid substitution through visual supervision up to 5 days and reassessing the handling of co-occurring substance use are seen as innovations with practical relevance. A minor benefit is the possibility to treat 10 instead of 3 patients under consultative care.

Conclusions The changed regulations are unlikely to be sufficient in attracting new physicians to offer opioid substitution treatment. Cooperations with pharmacies, care facilities and governmental institutions will become increasingly important. Support for independent physicians through substitution outpatient clinics and psychiatric outpatient clinics is necessary. The treatment of addiction should be given greater priority in medical education.

\section{Einleitung}

Die Opioidsubstitutionstherapie (opioid substitution treatment OST) ist die wichtigste Therapieoption zur Behandlung der Opioidabhängigkeit in Deutschland. Etwa die Hälfte aller Opioidabhängigen in Deutschland befinden sich jährlich in einer OST [1], die nachweislich den Gebrauch von illegalen Substanzen, das HIV- und Hepatitis-C-Risikoverhalten sowie die Todesraten durch OpioidÜberdosierungen reduziert, die soziale Integration fördert und die Beschaffungskriminalität verringert [2-8]. Trotz der sichtbaren individuellen und gesamtgesellschaftlichen Effekte der Behandlung limitieren strukturelle, insbesondere rechtliche Rahmenbedingungen die Ausweitung der OST $[9,10]$. Heute haben bereits viele substituierende ÄrztInnen das Renteneintrittsalter erreicht oder stehen kurz davor und es zeigen sich, insbesondere in ländlichen Gegenden, grundsätzliche Probleme, Nachfolgerlnnen für die Kassensitze zu finden $[11,12]$. Selbst bei Kassensitzübernahmen ist nicht gewährleistet, dass die Substitutionsbehandlung weitergeführt wird [13]. Nicht nur auf Seite der Behandelnden, auch patientenseitig steht die OST vor Herausforderungen. Mit zunehmendem Alter der PatientInnen zeigen sich Veränderungen hinsichtlich altersbedingter, chronischer und multipler Komorbiditäten $[14,15]$. Hieraus ergeben sich zukünftig neue Aufgaben für die OST, wie beispielsweise eine Ausweitung der Behandlung in Einrichtungen der ambulanten und stationären Pflege.

Um den Anforderungen einer zukünftigen Versorgung opioidabhängiger Menschen gerecht zu werden und letztendlich auch die Anzahl substituierender ÄrztInnen in Deutschland zu erhöhen, wurde im Mai 2017 die 3. Verordnung zur Änderung der Betäubungsmittel-Verschreibungsverordnung (3. BtMVVÄndV) verabschiedet [16]. Übergeordnete Ziele waren die Verbesserung der Rechtssicherheit für substituierende Ärztlnnen und die Anpassung der Durchführung von Substitutionsbehandlungen an den neusten wissenschaftlichen Stand sowie an praktische Erfordernisse. Dazu werden in Deutschland zukünftig die medizinisch-therapeutischen Aspekte der Substitutionsbehandlung ausschließlich durch die Richtlinie der Bundesärztekammer (BÄK) geregelt [17]. In die neue Richtlinienkompetenz der BÄK wurden im Wesentlichen die Therapieziele, die Voraussetzungen für die Einleitung und Fortführung einer Substitutionsbehandlung sowie die Erstellung eines Therapiekonzepts überführt. Die BÄK hatte dazu in ihrer „Richtlinie zur Durchführung der substitutionsgestützten Behandlung Opioidabhängiger" den aktuellen wissenschaftlichen Stand zur Opioidsubstitutionsbehandlung erarbeitet [18]. Die neuen Richtlinien sind in - Tab. 1 im Vergleich zu den zuvor gültigen dargestellt.

Mit diesen Änderungen der Betäubungsmittel-Verschreibungsverordnung (BtMVV) wurden die seit langem vertretenen Forderungen der Interessenvertreter weitgehend erfüllt. Die Studie von Schulte et al. befragte 2009 substituierende ÄrztInnen in Deutschland zu strukturellen Barrieren in der Substitutionsbehandlung. Jeweils etwa $80 \%$ sahen diese insbesondere durch mögliche rechtliche Konsequenzen bei Verstößen gegen die BtMVV sowie durch einen daraus resultierenden hohen Verwaltungsaufwand gegeben [19].

Um zu überprüfen, wie die Neuregelung zur substitutionsgestützten Behandlung opioidabhängiger Menschen in der Praxis 
- Tab. 1 Alte und neue Richtlinien zur Durchführung der Substitutionsbehandlung.

\begin{tabular}{|c|c|c|}
\hline & Alte Substitutionsrichtlinien & $\begin{array}{l}\text { Neue Substitutionsrichtlinien nach Inkrafttreten der } \\
\text { 3. BtMVVÄndV am 02.10.2017 }\end{array}$ \\
\hline Rechtssicherheit & $\begin{array}{l}\text { Alle Regelungen nur im Rahmen der Betäubungsmittel- } \\
\text { Verschreibungsverordnung (BtMVV). }\end{array}$ & $\begin{array}{l}\text { Regelungen, die ärztlich-therapeutische Bewertungen } \\
\text { betreffen, werden aus dem Rahmen der BtMVV in die } \\
\text { Richtlinienkompetenz der BÄK überführt. Verfolgungsmög- } \\
\text { lichkeiten durch die Justiz sind dadurch eingeschränkt. }\end{array}$ \\
\hline $\begin{array}{l}\text { Konsum weiterer } \\
\text { psychotroper } \\
\text { Substanzen } \\
\text { (Beikonsum) }\end{array}$ & $\begin{array}{l}\text { Es dürfen keine Substanzen gebraucht werden, deren } \\
\text { Konsum nach Art und Menge den Zweck der Substitution } \\
\text { gefährden. }\end{array}$ & $\begin{array}{l}\text { Der Beikonsum anderer psychotroper Substanzen und Alkohol } \\
\text { soll kontrolliert und während der Substitution berücksichtigt } \\
\text { und ggf. therapiert werden. }\end{array}$ \\
\hline $\begin{array}{l}\text { Take-Home, } \\
\text { Ausnahme vom } \\
\text { Sichtbezug }\end{array}$ & $\begin{array}{l}\text { Eine Take-Home-Verordnung von bis zu } 7 \text { Tagen ist } \\
\text { möglich und bis zu } 2 \text { Tagen zur Überbrückung bei } \\
\text { grundsätzlichem Sichtbezug. }\end{array}$ & $\begin{array}{l}\text { Eine Take-Home-Verordnung von bis zu } 30 \text { Tagen ist möglich } \\
\text { und bis zu } 5 \text { Tagen zur Überbrückung bei grundsätzlichem } \\
\text { Sichtbezug. }\end{array}$ \\
\hline Abstinenz & $\begin{array}{l}\text { Ziel der Substitutionstherapie ist die schrittweise } \\
\text { Wiederherstellung der Betäubungsmittelabstinenz. }\end{array}$ & $\begin{array}{l}\text { Eine Opioidabstinenz ist anzustreben, muss aber nicht erreicht } \\
\text { werden. Zeitliche Vorgaben bestehen nicht. }\end{array}$ \\
\hline $\begin{array}{l}\text { Psychosoziale } \\
\text { Begleitung (PSB) }\end{array}$ & $\begin{array}{l}\text { Substitution nur unter der Voraussetzung, dass eine } \\
\text { erforderliche PSB einbezogen wird. }\end{array}$ & $\begin{array}{l}\text { PSB soll weiterhin regelhaft empfohlen werden, ist aber keine } \\
\text { Voraussetzung mehr für die Substitutionsbehandlung. }\end{array}$ \\
\hline Konsiliararztmodell & $\begin{array}{l}\text { Suchtmedizinisch nicht qualifizierte Ärztlnnen dürfen bis } \\
\text { zu } 3 \text { PatientInnen mit Substitutionsmitteln unter der } \\
\text { Aufsicht suchtmedizinisch qualifizierter Ärztlnnen } \\
\text { (Konsiliarii) behandeln. }\end{array}$ & $\begin{array}{l}\text { Suchtmedizinisch nicht qualifizierte ÄrztInnen dürfen bis zu } 10 \\
\text { PatientInnen mit Substitutionsmitteln unter der Aufsicht } \\
\text { suchtmedizinisch qualifizierter ÄrztInnen (Konsiliarii) } \\
\text { behandeln. }\end{array}$ \\
\hline Synthetische Opioide & $\begin{array}{l}\text { Substitution ist die Anwendung eines ärztlich verschriebe- } \\
\text { nen Betäubungsmittels bei einer opiatabhängigen } \\
\text { Patientln. }\end{array}$ & $\begin{array}{l}\text { PatientInnen, die ausschließlich von synthetischen Opioiden } \\
\text { (z. B. Tilidin oder Tramadol) abhängig sind, können in die } \\
\text { Substitutionsbehandlung aufgenommen werden. }\end{array}$ \\
\hline $\begin{array}{l}\text { Abgabe des } \\
\text { Substitutionsmittels }\end{array}$ & $\begin{array}{l}\text { Das Substitutionsmittel ist Patientlnnen in einem } \\
\text { Krankenhaus oder in einer Apotheke oder in einer hierfür } \\
\text { von der zuständigen Landesbehörde anerkannten anderen } \\
\text { geeigneten Einrichtung oder, im Falle einer ärztlich } \\
\text { bescheinigten Pflegebedürftigkeit, bei einem Hausbesuch } \\
\text { zum unmittelbaren Verbrauch zu überlassen. }\end{array}$ & $\begin{array}{l}\text { Das Substitutionsmittel darf durch medizinisches, pharmazeu- } \\
\text { tisches oder pflegerisches Personal in einer stationären } \\
\text { Einrichtung der medizinischen Rehabilitation, einem } \\
\text { Gesundheitsamt, einem Alten- oder Pflegeheim, einem Hospiz } \\
\text { oder einer anderen geeigneten Einrichtung, die zu diesem } \\
\text { Zweck von der zuständigen Landesbehörde anerkannt sein } \\
\text { muss, abgegeben werden. }\end{array}$ \\
\hline
\end{tabular}

bewertet und angenommen wird, wurde von der Gesundheitsministerkonferenz der Länder einstimmig deren Evaluation beschlossen [20]. In diesem Artikel werden die Ergebnisse leitfadengestützter Interviews mit substituierenden ÄrztInnen in 4 deutschen Bundesländern vorgestellt. Die Bewertung und praktische Umsetzung der neuen Richtlinien zur Substitution, strukturelle Voraussetzungen der Substitutionsbehandlung sowie Vorstellungen für deren zukünftige Gestaltung standen im Fokus der Befragung.

\section{Methodik}

\section{Studiendesign}

Die vorliegende Untersuchung fand als Teilprojekt im Rahmen der vom Bundesministerium für Gesundheit (BMG) geförderten Evaluation der 3. Verordnung zur Änderung der BtMVV vom 22.5.2017 („Substitutions-Novelle“) - EVASUNO im Zeitraum von Dezember 2019 bis April 2020 statt. Das Evaluationskonzept der Gesamtstudie umfasst modular aufgebaute quantitative und qualitative $\mathrm{Er}$ hebungsstrategien sowie Sekundärdatenanalysen über einen Zeitraum von 3 Jahren. Hierbei werden mögliche Effekte aus Sicht der
PatientInnen sowie VersorgerInnen (ÄrztInnen, ApothekerInnen) untersucht. Ergänzend zu einer bundesweiten schriftlichen Befragung substituierender ÄrztInnen wurden in 4 ausgewählten Modellregionen vertiefende leitfadengestützte Interviews mit substituierenden ÄrztInnen geführt. Die Auswahl dieser Regionen erfolgte einerseits geografisch (städtisch versus ländlich) sowie gezielt nach besser und schlechter bzw. unterversorgten Gebieten. Als Modellregionen wurden Hamburg, Nordrhein-Westfalen (Kassenärztlicher Vereinigungs (KV)-Bezirk Nordrhein und KV-Bezirk Westfalen-Lippe), Sachsen und Bayern ausgewählt. Die Interviews wurden vor Ort in den Praxen der Teilnehmenden geführt. Die ÄrztInnen wurden über die Studie aufgeklärt, gaben ihr schriftliches Einverständnis zur Audioaufnahme und erhielten eine Aufwandsentschädigung. Ein positives Ethikvotum der Lokalen Psychologischen Ethikkommission am Zentrum für Psychosoziale Medizin (LPEK) des Universitätsklinikums Hamburg-Eppendorf liegt vor (LPEK-0084).

\section{Erhebungsinstrument}

Die Inhalte des Interviewleitfadens bezogen sich auf strukturelle Merkmale (ärztliches Fachgebiet, Qualifikation und Erfahrung, Anzahl Substituierter, Art der Praxis) sowie auf die in der 3. BtMVV- 
ÄndV beschlossenen Neuerungen (größere Rechtssicherheit, neue Bewertung des Beikonsums, veränderte Take-Home-Regelungen, Nutzung der Konsiliarregelung, Herausnahme der Erforderlichkeit psychosozialer Betreuungsmaßnahmen und Kooperation mit Apotheken sowie anderen Einrichtungen). Darüber hinaus enthielt der Leitfaden Fragen zur Kooperation und Vernetzung substituierender Ärztlnnen und möglichen Versorgungsengpässen in der Region.

\section{Stichprobe}

In den Modellregionen wurden insgesamt 31 Interviews mit substituierenden ÄrztInnen geführt. In NRW und Bayern lehnten insgesamt 5 der angefragten potenziellen InterviewpartnerInnen eine Teilnahme ab. Alle Interviewten verfügten über die Zusatzweiterbildung ,Suchtmedizinische Grundversorgung، oder die Fachkunde Sucht. In Hamburg wurden 5, in Nordrhein-Westfalen 12 (Westfalen-Lippe 6 Interviews, Nordrhein 6 Interviews), in Bayern 10 Interviews mit ÄrztInnen aus allen 7 Regierungsbezirken (Unterfranken, Schwaben, Oberpfalz, Oberfranken, Mittelfranken, Oberbayern, Niederbayern) und in Sachsen 4 Interviews durchgeführt. Um sicherzustellen, dass die Interviewten Einschätzungen zur Versorgungssituation in ihrer Region geben konnten, wurden keine substituierenden ÄrztInnen in die Befragung aufgenommen, die unter einer Konsiliarärztin oder einem Konsiliararzt tätig waren.

\section{Auswertung}

Die Interviews dauerten zwischen 60 und 90 Minuten. Die Aufnahmen wurden innerhalb von 5 Tagen transkribiert, anonymisiert und anschließend gelöscht. Auf wörtliche Zitate wird in dem vorliegenden Artikel verzichtet, um die Anonymität der interviewten ÄrztInnen zu wahren. Für den vorliegenden Artikel wurden die Transkripte von 2 ForscherInnen entsprechend der Leitfadenbereiche gruppiert und ausgewertet. Im Rahmen des vollständigen Durchlaufs des Originalmaterials wurden relevante Textbestandteile identifiziert, extrahiert und Unterkategorien zur Bewertung und praktischen Relevanz der Änderungen der 3. BtMVVÄndV sowie der strukturellen Bedingungen zugeordnet.

\section{Ergebnisse}

Es wurden Interviews mit substituierenden Ärztlnnen in unterschiedlichen Versorgungssettings geführt ( $>$ Tab. 2). Das Durchschnittsalter lag bei 58,5 Jahren, 77,4\% waren männlich und 54,8\% konsiliarisch tätig. Neben in privater Praxis niedergelassenen ÄrztInnen haben sich auch angestellte ÄrztInnen sowie ÄrztInnen aus Substitutionsambulanzen, PIAs und Medizinischen Versorgungszentren (MVZ) an den Interviews beteiligt. Dementsprechend hoch war die Spannweite der substituierten PatientInnen pro Einrichtung (35-800). Ebenfalls wurden große Unterschiede in der Größe des Versorgungsgebiets der Substitutionseinrichtungen gefunden. Die maximale Entfernung zwischen Wohnort der PatientInnen und den Substitutionspraxen betrug zwischen 10 und $170 \mathrm{~km}$.

\section{Bewertung der Änderungen der 3. BtMVVÄndV}

Die Änderungen der 3. BtMVVÄndV wurden von den meisten Interviewten positiv bewertet. Bezogen auf die praktische Relevanz der Änderungen bestehen Unterschiede zwischen den einzelnen Bundesländern ( $\triangleright$ Tab. 3).

\section{Rechtssicherheit}

Die größere Rechtssicherheit durch die Überführung der ärztlichtherapeutischen Bereiche in die Richtlinienkompetenz der BÄK wird von fast allen Interviewten positiv bewertet. In den Bundesländern Sachsen und Bayern muss sich die Änderung erst noch bewähren. In Bayern ist die ,Angst vor dem Staatsanwalt' bei vielen substituierenden ÄrztInnen weiterhin spürbar, da jeder jemanden kennt, der in der Vergangenheit schon einmal große rechtliche Probleme hatte. Unter den StaatsanwältInnen, RichterInnen aber auch unter den Mitgliedern der Ärztekammern gäbe es BefürworterInnen und GegnerInnen der Substitution. Es müsse sich erst noch zeigen, ob Formfehler oder ärztliche Entscheidungen tatsächlich vor dem Berufsgericht ohne direkte strafrechtliche Konsequenzen diskutiert werden.

\section{Konsum weiterer psychotroper Substanzen (Beikonsum)}

Die rechtlich abgesicherte Möglichkeit, die Substitution auch bei fortgesetztem Beikonsum anderer psychoaktiver Substanzen fortführen zu können, entspricht laut Interviewten einer guten medizinischen Praxis der Suchtbehandlung, zumal die meisten PatientInnen einen polyvalenten Konsum praktizieren. Die Reduktion des Beikonsums bleibt ein zentrales Thema in der suchtmedizinischen Behandlung, wird jedoch realistisch über einen langen Zeitraum gedacht. Einigen PatientInnen falle es somit leichter bzw. werde es erstmals möglich, ihren Beikonsum ehrlich anzugeben, ohne einen Ausschluss aus der Behandlung befürchten zu müssen. Auf diesem Hintergrund seien Gespräche über problematischen Beikonsum in einer offeneren Atmosphäre durchführbar. Während in Sachsen kristallines Methamphetamin als ein zentrales Thema genannt wurde, was zu großen, in einigen Fällen irreversiblen gesundheitlichen Schäden der Substituierten und zu einer Steigerung der Aggressivität geführt hat, spielen in Bayern eher synthetische Cannabinoide (,Kräutermischungen') als Cannabisersatz eine Rolle. Alkohol, Benzodiazepine, Z-Substanzen und Pregabalin wurden als weitere Substanzen genannt. Bezogen auf Pregabalin ist unklar, ob der Beikonsum dieser Substanz in der Zukunft eher ab- oder zunehmen wird. Positiv angemerkt wurde, dass es mittlerweile aussagekräftige und fälschungssichere, jedoch teurere Analysemethoden wie beispielweise Speicheltests gibt. Sie könnten eine Alternative zu der Urinabgabe unter Sicht darstellen.

\section{Take-Home und Ausnahme vom Sichtbezug}

Die Möglichkeit einer Take-Home-Verordnung von bis zu 30 Tagen und die Ausnahme vom Sichtbezug von 5 Tagen werden als eine Erleichterung im Praxisalltag gewertet. Während zuvor für Arbeitseinsätze außerhalb des Wohnorts, Urlaube oder auch kurze Verwandtenbesuche ,kreative und z. T. aufwendige Lösungen gefunden werden mussten, sind nunmehr Ausnahmen leichter möglich. Allerdings sind die substituierenden ÄrztInnen bei einer TakeHome-Verschreibung von 3 Wochen oder 30 Tagen zurückhaltend und setzen diese nur ausnahmsweise bei sehr stabilen PatientInnen ein. Die PatientInnen kennen zumeist diese neue Verschrei- 
- Tab. 2 Charakteristika der in den Modellregionen interviewten ÄrztInnen ( $N=31)$.

\begin{tabular}{|c|c|}
\hline Männlich, N (\%) & $24(77,4 \%)$ \\
\hline \multirow[t]{2}{*}{ Alter in Jahren, $M(S D)$} & $58,5(8,1)$ \\
\hline & (39-72 Jahre) \\
\hline \multirow[t]{2}{*}{ Jahre in Substitution tätig, $M(S D)$} & $20,4(8,4)$ \\
\hline & (3-30 Jahre) \\
\hline \multirow[t]{2}{*}{ Anzahl substituierter PatientInnen, M (SD) } & $209,2(191,6)$ \\
\hline & (35-800 PatientInnen) \\
\hline \multicolumn{2}{|l|}{ Facharztrichtung, $\mathrm{N}(\%)$} \\
\hline Allgemeinmedizin und/oder Innere Medizin & $25(80,6 \%)$ \\
\hline Psychiatrie und Psychotherapie/Neurologie & $6(29,4 \%)$ \\
\hline \multicolumn{2}{|l|}{ Art der Praxis, $N(\%)$} \\
\hline Einzelpraxis/Gemeinschaftspraxis/Praxisgemeinschaft & $23(74,2 \%)$ \\
\hline Substitutionsambulanz & $3(9,7 \%)$ \\
\hline PIA & $2(6,4 \%)$ \\
\hline MVZ & $3(9,7 \%)$ \\
\hline \multicolumn{2}{|l|}{ Praxisorganisation, $\mathrm{N}(\%)$} \\
\hline Praxisbetrieb und Substitution räumlich getrennt & $8(25,8 \%)$ \\
\hline Praxisbetrieb und Substitution räumlich nicht getrennt & $16(51,6 \%)$ \\
\hline kein weiterer Praxisbetrieb, ausschließlich Substitution & $7(22,6 \%)$ \\
\hline \multirow[t]{2}{*}{ Größe des Einzugsgebietes in km, M (SD) } & $57,3(45,8)$ \\
\hline & $(10-170 \mathrm{~km})$ \\
\hline Konsiliarisch tätig, N (\%) & $17(54,8 \%)$ \\
\hline
\end{tabular}

- Tab. 3 Bewertung und praktische Relevanz der 3. BtMVVÄndV in den Bundesländern Hamburg (HH), Sachsen (SN), Nordrhein-Westfalen (NRW) und Bayern (BY).

\begin{tabular}{|c|c|c|c|c|c|c|c|c|}
\hline \multirow{2}{*}{$\begin{array}{l}\text { Neuerungen der 3. BtMV- } \\
\text { VÄndV }\end{array}$} & \multicolumn{4}{|c|}{ Bewertung * } & \multicolumn{4}{|c|}{ Praktische Relevanz ${ }^{*}$} \\
\hline & HH & NRW & SN & BY & HH & NRW & SN & BY \\
\hline $\begin{array}{l}\text { Größere Rechtssicherheit, } \\
\text { Bewertung durch BÄK }\end{array}$ & ++ & + & ++ & + & $+1-$ & + & $+1-$ & + \\
\hline Neue Bewertung Beikonsum & ++ & ++ & ++ & ++ & - & $+/ 0$ & + & + \\
\hline $\begin{array}{l}\text { Take-Home: max. } 30 \text { Tage/ } \\
\text { Ausnahme vom Sichtbezug: } 5 \\
\text { Tage }\end{array}$ & ++ & ++ & ++ & ++ & ++ & + & + & ++ \\
\hline $\begin{array}{l}\text { Keine zeitliche Vorgabe für das } \\
\text { Erreichen einer Opioidabstinenz }\end{array}$ & ++ & ++ & ++ & ++ & - & -- & -- & + \\
\hline $\begin{array}{l}\text { Herausnahme der Erforderlichkeit } \\
\text { von PSB }\end{array}$ & ++ & ++ & $+1-$ & $+1-$ & ++ & - & $+1-$ & - \\
\hline $\begin{array}{l}\text { Konsiliarbehandlung von } 10 \\
\text { PatientInnen möglich }\end{array}$ & -- & -- & - & - & -- & -- & - & -- \\
\hline $\begin{array}{l}\text { Substitution von PatientInnen mit } \\
\text { Abhängigkeit von synthetischen } \\
\text { Opioiden }\end{array}$ & + & + & $+1-$ & ++ & -- & $+/-$ & -- & -- \\
\hline
\end{tabular}


bungsmöglichkeit, drängen aber nicht stärker als vorher auf eine Take-Home-Mitgabe. Häufiger werden 14-Tage-Rezepte ausgegeben, oft in Form eines so genannten Mischrezepts. Das bedeutet, dass die PatientInnen in der Apotheke jeweils für eine Woche das Substitut mit nach Hause bekommen und es zwischenzeitlich einmal unter Sicht in der Apotheke einnehmen müssen. Eine verlängerte Take-Home-Mitgabe machen die BehandlerInnen auch von der Dosis und der Art des Substitutionsmittels abhängig, da sie nicht möchten, dass die PatientInnen einen größeren Bestand zu Hause lagern. Ferner spielt die Art und Menge des Beikonsums eine Rolle bei der Entscheidung für eine Take-Home-Verschreibung. Die erweiterte Take-Home-Mitgabemöglichkeit wird auch unter dem Aspekt der Teilhabe der PatientInnen am sozialen Leben positiv bewertet.

\section{Keine zeitliche Vorgabe für das Erreichen einer Opioidabstinenz}

Auch diese Neuerung wird positiv bewertet, da sich die BehandlerInnen nunmehr weder der KV, noch der Krankenkasse oder dem Medizinischen Dienst der Krankenversicherung (MDK) gegenüber rechtfertigen müssen. Das mögliche Therapieziel Abstinenz, allerdings ohne eine zeitliche Vorgabe, behalten die BehandlerInnen dennoch bei den meisten PatientInnen im Auge und regen bei langfristig stabilen PatientInnen immer wieder Abdosierungsversuche an. Allerdings wurde deutlich, dass die allermeisten möglicherweise eine lebenslange Substitutionsbehandlung benötigen.

\section{Psychosoziale Begleitung (PSB)}

Grundsätzlich wird begrüßt, dass eine Substitutionsbehandlung nicht mehr an die PSB gebunden ist. Die erforderlichen Bescheinigungen beizubringen habe viel Kapazität gebunden, zumal es immer auch PatientInnen gab, die nicht zur Teilnahme an einer PSB zu bewegen waren bzw. deren PSB-Teilnahme nicht notwendig war. Die ÄrztInnen mussten finanzielle Einbußen hinnehmen, wenn ihnen die entsprechenden Vergütungsziffern abgezogen wurden. Es sei bei sehr stabilen PatientInnen absurd gewesen, eine entsprechende Bescheinigung beibringen zu müssen.

Selbstkritisch wird angemerkt, dass weniger ,Drängeln` auch dazu führen kann, dass PatientInnen, die vielleicht eine PSB nötig hätten, in einzelnen Fällen keine PSB in Anspruch nähmen. Eine begleitende PSB halten alle ÄrztInnen allerdings für sinnvoll, vor allem bei neu eingestellten PatientInnen sowie anlassbezogen in bestimmten Lebensphasen oder persönlichen Krisen. Die meisten der interviewten ÄrztInnen verfahren wie bisher; einige verlangen weiterhin von allen Patientlnnen eine Bescheinigung.

\section{Konsiliararztmodell}

Die nun mögliche Aufstockung der PatientInnenzahl auf 10 wird von fast allen substituierenden Ärztlnnen für eine Überforderung gehalten, sowohl für die unter Supervision substituierenden ÄrztInnen, als auch für den Konsiliarius. Das Konsiliararztmodell wird von den meisten Interviewten grundsätzlich in Frage gestellt, ist aber im ländlichen Bereich oft die einzige Möglichkeit, substituierte PatientInnen wohnortnah zu versorgen. Eine an den Interviews teilnehmende Substitutionspraxis ist Konsiliarius für sehr viele ÄrztInnen, von denen inzwischen 2 auf 10 PatientInnen aufgestockt haben. Dies kann aber nur durch eine sehr intensive Betreuung der ÄrztInnen, teilweise vor Ort, realisiert werden.

\section{Opioide anstatt Opiate}

PatientInnen mit einer Abhängigkeit von (halb-)synthetischen Opioiden rechtlich abgesichert substituieren zu können, wird einheitlich begrüßt. Für die meisten Interviewten ist dies derzeit allerdings ohne große praktische Relevanz, da die bestehenden Regularien in Deutschland bisher eine Situation wie in den USA mit einer sehr großen Anzahl von PatientInnen mit einer iatrogenen Opioidabhängigkeit verhindert haben. Nur wenige ÄrztInnen berichten von erfolgreichen Behandlungen. Vielen Interviewten ist die Vorstellung unangenehm, diese PatientInnen dem Schema einer Substitutionstherapie unterwerfen zu müssen. Eine Zusammenarbeit mit Schmerztherapeutlnnen wäre wünschenswert.

\section{Abgabe des Substitutionsmittels}

Um die wohnortnahe Versorgung der SubstitutionspatientInnen zu verbessern, können Substitutionsmittel in Zukunft in stationären Einrichtungen der medizinischen Rehabilitation, Gesundheitsämtern, Alten- oder Pflegeheimen zum unmittelbaren Gebrauch abgegeben werden, sofern die substituierende Ärztln mit diesen Einrichtungen eine Vereinbarung getroffen hat. Diese neuen Möglichkeiten werden begrüßt und als Erleichterung gesehen. In der Vergangenheit haben die substituierenden ÄrztInnen kranke PatientInnen teilweise täglich im Hausbesuch selbst versorgt. Dies sei bei Einzelfällen noch leistbar gewesen, aber vor dem Hintergrund einer alternden Klientel und in Erwartung einer zunehmenden Pflegebedürftigkeit nicht mehr durchführbar.

Die meisten Interviewten haben sich, wenn die Notwendigkeit bestand, aktiv auf die Suche nach stationären oder ambulanten Pflegediensten begeben mit bisher durchweg guten Erfahrungen, v. a. mit ambulanten Pflegediensten. Proaktiv wurden Pflegedienste in die Qualitätszirkel eingeladen, in Ausnahmefällen haben Pflegedienste ihrerseits ihr Interesse bekundet und um Informationen gebeten. Stationäre Pflegeeinrichtungen erscheinen den Interviewten eher als eine ,Black Box und es sei unklar, wie gut die Pflegekräfte ausgebildet sind. Dies wird auf dem Hintergrund, dass die ÄrztInnen immer in der Verantwortung bleiben, kritisch gesehen, zumal die Vertragslage vielfach ungeklärt ist. Auch in der Palliativpflege seien die betreuenden Ärztlnnen anfangs nicht auf die hohen Opioiddosen eingestellt. Rehabilitationseinrichtungen, in denen die Substitutionsbehandlung problemlos weitergeführt wird, sind äußert selten. Es würde nach wie vor erwartet, dass PatientInnen vor der Rehabilitationsbehandlung entweder abdosiert oder entgiftet werden.

\section{Merksatz}

Die geänderte Take-Home-Regelung ist für die BehandlerInnen in der Praxis am wichtigsten.

\section{Strukturelle Voraussetzungen}

Die Größe des Einzugsgebiets einer substituierenden Einrichtung ist nicht nur davon abhängig, ob sich diese in einem großstädtischen oder ländlichen Bereich befindet. Die PatientInnen fahren auch aus dem Umland in die Großstädte, wenn die Versorgung 
nicht gewährleistet ist. Anzunehmen ist auch, dass die Lage der Praxen, ihre Erreichbarkeit sowie ihre Hoch- bzw. Niedrigschwelligkeit dabei eine große Rolle spielt.

\section{PsychiaterInnen}

In allen Modellregionen besteht ein Mangel an psychiatrischen Praxen, die substituierte PatientInnen (mit-)behandeln. Substituierende somatische ÄrztInnen verfügen i. d. R. über eine Expertise in der Behandlung psychischer Störungen, die auf viel Erfahrung beruht. Die Situation wird dann als zufriedenstellend erlebt, wenn wenigstens eine psychiatrische Praxis oder psychiatrische Ambulanz vor Ort zur Mitbehandlung bzw. Kooperation bereit ist. Für substituierende ÄrztInnen ist es außerordentlich wichtig, dass psychiatrische Abteilungen der Bezirkskrankenhäuser/Universitätskliniken bereit sind, psychiatrische Notfälle aufzunehmen und zeitnah Teilentgiftungen durchführen. Dies ist nicht überall gegeben und teilweise rückläufig. PsychotherapeutInnen stehen fast durchgehend nicht für diese Klientel zur Verfügung. Eine bessere Kooperation wird angemahnt. Eine hausärztliche Betreuung somatischer Erkrankungen, auch im Hinblick auf die zunehmend älter werdenden PatientInnen, solle stets gewährleistet sein.

\section{Apotheken}

Die Zusammenarbeit mit Apotheken wird in einem unterschiedlichen Ausmaß benötigt und ist für Praxen mit einer eigenen Vergabe der Substitutionsmittel nicht vorrangig. Die Zusammenarbeit wird durchgehend als sehr gut bewertet und die Apotheken seien motiviert und verantwortungsvoll im Umgang mit den PatientInnen. Praxen mit einem großen Einzugsgebiet, wie in Sachsen oder Bayern, sind in einem hohen Maße auf die Zusammenarbeit mit Apotheken, welche die Sichtvergabe durchführen, angewiesen. Oftmals ist von den substituierenden ÄrztInnen Überzeugungsarbeit zu leisten, was auch eine persönliche Kontaktaufnahme mit den ApothekerInnen in der Apotheke vor Ort einschließen kann. Es gibt aber weiterhin Gebiete, in denen keine einzige Apotheke zur Substitutionsvergabe unter Sicht bereit ist.

Die Apotheken kämen ihrer monatlichen Informationspflicht über die Nachweisführung der Sichtvergabe sehr gut nach und genaue Informationen über die Vergabe können bei Bedarf abgerufen werden. Die neuen Apothekenverträge der Bundesapothekerkammer (ABDA) ${ }^{1}$ zur Überlassung von Substitutionsmitteln zum unmittelbaren Gebrauch werden jedoch selten angewandt und als zu umfangreich eingestuft. Zur Anwendung kommen eigene Verträge, was aber als unbefriedigend bewertet wird. Weitgehend einig sind sich die substituierenden ÄrztInnen dahingehend, dass Apotheken für die Sichtvergabe der Substitutionsmittel entlohnt werden sollten, da auch Hygienevorschriften, Geräte, Vergabetresen usw. finanziert werden müssten.

\section{Kooperationen und Vernetzungen}

Es wurden in der Vergangenheit viele formale Netzwerke aufgebaut, dazu gehören ,Runde Tische“ und Qualitätszirkel, teilweise über Bezirksgrenzen oder Bundeslandgrenzen hinaus. Hier kom-

\footnotetext{
https://www.abda.de/fuer-apotheker/qualitaetssicherung/leitlinien/leitlinien-und-arbeitshilfen/
}

men unterschiedliche Professionen zusammen; neben den substituierenden ÄrztInnen auch ApothekerInnen, VertreterInnen von KVen, Krankenkassen, Polizei, Staatsanwaltschaft, PSB, VertreterInnen von Kliniken, Gesundheitsämtern, Justizvollzugsanstalten, Bewährungshilfen und Pflegediensten. ÄrztInnen, die unter konsiliarischer Betreuung arbeiten, sind zur Teilnahme nur sehr eingeschränkt zu motivieren. Im Laufe der Zeit sind auch stabile informelle Netzwerke entstanden. Übereinstimmung besteht darin, dass niedergelassene ÄrztInnen nicht alle opioidabhängigen PatientInnen substituieren können. Es kommt immer wieder vor, dass einzelne PatientInnen in einer Hausarztpraxis nicht mehr tragbar sind. Hierfür bietet es sich an und wird auch praktiziert, sehr problematische PatientInnen an Substitutionsambulanzen oder Schwerpunktpraxen abzugeben und dafür von diesen stabile PatientInnen aufzunehmen. Wenn keine Ambulanzen oder Schwerpunktpraxen vorhanden sind, müssten diese PatientInnen auf große Städte ausweichen, teilweise auch über Bundeslandgrenzen hinweg (z. B. fahren PatientInnen aus Unterfranken nach Offenbach oder Frankfurt am Main). Durchaus häufig sind Netzwerke in NRW und Bayern, in denen sich substituierende ÄrztInnen zu einer zentralen Wochenendvergabe zusammengeschlossen haben. In ländlichen Gebieten ist dies allerdings nicht möglich, da die PatientInnen dann unter Umständen am Wochenende noch größere Enfernungen bewältigen müssten.

\section{Merksatz}

Für substituierende ÄrztInnen können Qualitätszirkel ein guter Ausgangpunkt für weitere Vernetzungstätigkeiten wie eine gemeinsame Wochenendvergabe sein.

\section{Finanzen}

Die finanzielle Vergütung wird von den meisten ÄrztInnen mit Einschränkungen als ausreichend bis gut eingeschätzt. Allerdings wird der Aufwand für ,schwierige ' PatientInnen nicht entsprechend abgebildet. Gespräche mit den PatientInnen würden weiterhin nicht ausreichend honoriert. Die Interviewten gehen davon aus, dass die finanziellen Regelungen die Take-Home-Vergabe nicht ermutigen und somit die Teilhabemöglichkeit der PatientInnen erschweren. Nach wie vor ist der Verdienst am höchsten, wenn die Vergabe des Substitutionsmittels täglich in den Praxen stattfindet. Auch bei längeren Take-Home-Zeiten bleiben der grundsätzliche Substitutionsaufwand und die Verantwortung hoch. Ob über eine bessere Finanzierung der Substitutionsbehandlung weitere Ärztlnnen gewonnen werden können, wird unterschiedlich eingeschätzt und mehrheitlich mit Skepsis betrachtet. Einzelne Gebiete könnten aber durchaus profitieren.

\section{Sicherung der Substitution}

Die Substitution brauche weitere, kontinuierliche politische Aufmerksamkeit. Die 3. BtMVVÄndV sei ein wichtiger Schritt in die richtige Richtung, werde aber ohne flankierende Maßnahmen an der drohenden Unterversorgung mit niedergelassenen ÄrztInnen grundlegend wenig ändern. Ohne den Rückhalt von Bezirkskliniken und angegliederten Substitutionsambulanzen bei der Versorgung von PatientInnen mit einer gravierenden psychiatrischen Ko- 
morbidität, geringer Adhärenz oder Verhaltensauffälligkeiten wie stark forderndes oder aggressives Auftreten ist zweifelhaft, ob neue niedergelassene Ärztlnnen gewonnen werden können. Positiv hervorgehoben wird der Pakt für Substitution in Baden-Württemberg. Der Wunsch berenteter SubstitutionsärztInnen, weiterhin in der Substitution aktiv zu bleiben, soll formal erleichtert werden. Auch wenn dies langfristig den Mangel an jüngeren substituierenden ÄrztInnen nicht ausgleichen könne, seien diese ÄrztInnen an einigen Standorten wichtig für die Aufrechterhaltung der Substitution. Zudem verfügen sie über sehr viel Erfahrung und eine hohe Motivation.

Obgleich flächendeckend der Wunsch geäußert wird, dass mehr PIA, Substitutionsambulanzen und Krankenhäuser möglichst niedrigschwellig substituieren mögen, so sollte doch der Fokus weiter auf niedergelassenen ÄrztInnen bleiben. Es sollte vermieden werden, dass nur eine reine Suchtbehandlung stattfindet und weitere Erkrankungen möglicherweise nicht diagnostiziert und behandelt würden. Gleichzeitig erscheint jedoch auch eine Zentrierung sinnvoll, um die Qualität der Substitutionsbehandlung zu garantieren und ggf. zu steigern. Daher wird auch das Konsiliararztmodell überwiegend sehr kritisch gesehen. Substitutionsbusse könnten ländliche Gebiete abdecken, aber auch hierfür fehle das Personal. Stärker in den Fokus sollten die Medizinischen Fachangestellten treten, denen eine große Bedeutung für die Vergabe zukommt. Die Dokumentation sollte weiter verschlankt werden und bei Fehlern, auch beim Ausfüllen von Rezepten, den ÄrztInnen nicht per se eine Absicht unterstellt und diese finanziell geahndet werden. Weitere Anstrengungen sollten unternommen werden, um der Stigmatisierung auf allen Ebenen, auch in Krankenhäusern, entgegenzuwirken.

Für die Interviewten ist es sehr wichtig, die Suchtbehandlung und speziell die Substitution stärker in die Ausbildung der MedizinerInnen zu integrieren. So sieht die Weiterbildungsordnung im Bereich Psychiatrie eine Tätigkeit im Suchtbereich vor. Das sollte konsequent umgesetzt werden. In den letzten Jahren sei viel Werbung für HausärztInnen gemacht worden, v. a. im ländlichen Bereich. Teilweise seien Erfolge spürbar, so würden Hausarztpraxen wieder besetzt und Lehrarztpraxen bekommen verstärkt Anfragen nach Ausbildungsplätzen. Dieses Zeitfenster müsse genutzt werden, da sich die ÄrztInnen nach Abschluss der fachärztlichen Ausbildung auf einen Bereich der ärztlichen Tätigkeit festgelegt haben. So sollte die Zusatzweiterbildung ,Suchtmedizinische Grundversorgung' schon vor dem Facharzt besucht und abgeschlossen werden können. Der Kurs selbst sei häufig allerdings sehr theoretisch, würde nicht spezifisch auf die tatsächlichen Anforderungen einer Substitutionsbehandlung vorbereiten. Zudem wäre es wichtig zu betonen, dass die praktische Arbeit Freude bereitet, weil man Erfolge sieht und interessante Menschen mit vielfältigen Lebenserfahrungen über eine lange Zeiträume begleitet und sein gesamtes medizinisches Wissen anwenden und erweitern könne. Jungen KollegInnen sollten die Vorteile bewusst gemacht werden, die auch darin bestehen können, in einem Verbund zu arbeiten und engagierte AnsprechpartnerInnen zu haben.

\section{Diskussion}

Diese Studie untersuchte, wie die im Oktober 2017 in Kraft getretenen Änderungen der BtMVV von substituierenden ÄrztInnen ein- geschätzt werden und welche Relevanz sie für den Substitutionsalltag sowie für die Rahmenbedingungen der Substitution in Deutschland haben. Es konnten substituierende Ärztlnnen aus unterschiedlichen Versorgungssettings und Praxisformen interviewt werden. Grundsätzlich werden die Änderungen der BtMVV positiv bewertet und Regularien, die bis dahin stets als hemmend und sogar bedrohlich genannt wurden, scheinen nun überwunden $[19,21]$. Das bundesdeutsche Betäubungsmittelgesetz wird nicht in Frage gestellt, hat es doch nach Meinung der Interviewten eine ,Opioidwelle', wie aus den USA bekannt, in Deutschland verhindert [22-24].

Die Änderungen der BtMVV wurden als erleichternd und praxisrelevant empfunden. Dazu gehören die Ausdehnung der TakeHome-Verordnung auf 30 Tage und die Ausnahme vom Sichtbezug auf 5 Tage sowie der veränderte Umgang mit Beikonsum. Ein wesentlicher Bestandteil der 3. BtMVVÄndV ist die Überführung der ärztlich-therapeutischen Sachverhalte in die Richtlinienkompetenz der BÄK. Die Landesärztekammern sind nun bei eventuellen Verstößen gegen die ärztlichen Berufspflichten im Rahmen der Substitutionsbehandlung die ersten Ansprechpartner. Während in Hamburg und Nordrhein-Westfalen schon vorher weniger Rechtsunsicherheit bestand, fällt in Bayern die tiefe Skepsis hinsichtlich der Kontrollbehörden auf. Hier muss sich die Änderung in der Praxis erst bewähren. Politische Entscheidungsträger auf Landesebene, sowie KVen und Ärztekammern müssten deutlich machen, dass die Substitution im Rahmen der vorliegenden Verordnungen gewollt ist und sicherstellen, dass z. B. Staatsanwälte oder Gesundheitsämter auch in kleineren Städten entsprechend verfahren. Die bürokratischen Anforderungen bleiben allerdings weiterhin hoch und sind fehleranfällig. Neben den Anklagen und Verurteilungen von substituierenden ÄrztInnen, die in der Vergangenheit mediale Aufmerksamkeit erlangten [10], sind die BehandlerInnen weiter von Regresszahlungen an Krankenkassen bei Bagatellfehlern bedroht.

Eine Möglichkeit, das Fehlen von substituierenden Hausarztpraxen auszugleichen, wäre eine weitere Zentrierung auf größere Praxen bzw. Versorgungszentren oder Ambulanzen. Die Unterversorgung in ländlichen Gebieten kann damit jedoch nicht verhindert werden, da solche Versorgungsmöglichkeiten eher im städtischen Bereich oder Ballungsräumen entstehen dürften. Die wohnortnahe Substitution in eher ländlichen Gegenden würde dadurch erschwert und die bisherige Vielfalt unterschiedlicher Substitutionspraxen langfristig in Frage gestellt. Auch wünschen nicht alle SubstitutionspatientInnen eine Behandlung in spezialisierten Praxen. Das Konsiliararztmodell ist für einige Gebiete unverzichtbar, kann aber eine drohende Unterversorgung von OpioidpatientInnen mit einem Substitutionswunsch nicht verhindern. Auch wenn $22 \%$ der ÄrztInnen im Rahmen der Konsiliarregelung unter suchtmedizinisch qualifizierten KonsiliarärztInnen substituieren, versorgt diese Gruppe nach den Angaben der Bundesopiumstelle lediglich $1 \%$ aller substituierten PatientInnen [25].

Merksatz

Quo vadis: Zentrierung der Substitutionsbehandlung auf wenige große Ambulanzen oder Erhalt der Vielfalt von Substitutionsangeboten? 
Der Wert einer gegenseitigen Vernetzung wurde in der vorliegenden Untersuchung sehr häufig betont, wie schon in der Studie von Schulte et al. dargestellt [19]. Eine praxisnahe Weiterbildung und die kollegiale Unterstützung bei ,schwierigen“ PatientInnen trägt dazu bei, dass ÄrztInnen eine Substitutionsbehandlung beginnen oder laufende Behandlungen nicht vorzeitig beenden [26]. Viele der interviewten substituierenden Ärztlnnen berichten über gut funktionierende lokale Verbünde zur gegenseitigen Unterstützung. Gute Kooperationen mit weiteren, an der Substitution beteiligten Professionen werden als hilfreich und unterstützend erlebt. Hervorzuheben ist die durchgehend gute Zusammenarbeit mit Apotheken, ohne deren Unterstützung eine annähernd flächendeckende Versorgung von substituierten PatientInnen nicht gegeben wäre. Auch die PSB wird weiter als ein unverzichtbarer Bestandteil der Substitutionsbehandlung betrachtet.

Eine erhöhte Prävalenz psychischer Komorbidität bei substituierten Opioidabhängigen ist hinlänglich bekannt [14, 15, 27]. In der PREMOS-Studie waren mit 38,4\% Depressionen die häufigsten Einzeldiagnosen gefolgt von Persönlichkeitsstörungen, Angststörungen und Schlafstörungen [28]. Die Studie von Lieb und Kolleglnnen konnte zudem zeigen, dass Substituierte in größeren Behandlungseinrichtungen mit mehr PatientInnenkontakten deutlich mehr psychische Erkrankungen aufwiesen [29]. Psychisch stark auffällige substituierte PatientInnen können oftmals nur unzureichend in niedergelassenen Hausarztpraxen versorgt werden. Die interviewten niedergelassenen ÄrztInnen wünschen sich daher die Möglichkeit, diese PatientInnen an Schwerpunktpraxen oder Ambulanzen ,im Tausch“ gegen stabilere Substituierte überweisen zu können. Der Mangel an PsychiaterInnen unter den substituierenden FachärztInnen in Deutschland ist offensichtlich [19,21]. Substituierte Opioidabhängige weisen jedoch nicht nur psychische Komorbiditäten auf, sondern sind häufig auch somatisch hoch belastet und Erkrankungen werden mit dem steigenden Alter der Substituierten zunehmen. Um diese PatientInnen ganzheitlich medizinisch versorgen zu können, ist die Zusammenarbeit unterschiedlicher medizinischer Fachrichtungen notwendig. Die mangelnde Bereitschaft von Psychiaterlnnen, diese Patientenklientel (mit) zu behandeln sowie lange Wartelisten, aber auch die Vorbehalte in Krankenhäusern und psychiatrischen Kliniken gegenüber diesen PatientInnen, erschwert die Arbeit der substituierenden ÄrztInnen. In ihrem Positionspapier hat die Deutsche Suchtgesellschaft den Abbau von Zugangsschwellen für substituierte OpioidpatientInnen in Angeboten ambulanter, teilstationärer und stationärer Rehabilitationseinrichtungen gefordert [30]. Älter werdende SubstitutionspatientInnen erfordern eine zunehmende Zusammenarbeit von substituierenden Ärztlnnen mit ambulanten und stationären Pflegediensten sowie Hospizen. Es bietet es sich an, Schulungsmaterialien zentral zu erarbeiten und gezielte Fortbildungsangebote vorzuhalten.

Die Stigmatisierung von Opioidabhängigen, aber auch von Menschen mit anderen Abhängigkeitserkrankungen, ist gesellschaftlich nicht überwunden und das allgemeine Krankheitsverständnis von Abhängigkeit hat moralische Zuschreibungen und Schuldzuweisungen (,schwacher Wille‘) nicht gänzlich ersetzt. Medizinerlnnen außerhalb der Substitution treten abhängigen Menschen allzu häufig mit einem Laienverständnis von Sucht und weniger mit pro- fessionellem Wissen gegenüber. Dies mag u. a. der Tatsache geschuldet sein, dass gut integrierte, unauffällige Opioidsubstituierte nicht wahrgenommen werden, wohingegen ,schwierige PatientInnen` auffällig sind und den alltäglichen Medizinbetrieb belasten oder sogar dominieren können.

Der Zeitpunkt der durchgeführten Interviews fiel, mit Ausnahme eines telefonischen Gesprächs im April, knapp vor den Beginn der von der Bundesregierung beschlossenen Beschränkungen sozialer Kontakte aufgrund der COVID-19-Pandemie. SubstitutionspatientInnen gehören aufgrund ihrer häufig bestehenden Komorbiditäten der COVID-19-Risikogruppe an. Gleichzeitig muss die (tägliche) Versorgung mit Substitutionsmedikamenten gewährleistet bleiben. Im Falle von Schließungen von Substitutionseinrichtungen stünden alternative Vergabestellen und Behandlungszentren kaum zur Verfügung [31]. Das im März erlassene ,Gesetz zum Schutz der Bevölkerung bei einer epidemischen Lage von nationaler Tragweite‘ ermächtigte das BMG Ausnahmen von Regelungen des BtMG und der BtMVV in der Vergabe- und Verordnungspraxis von Substitutionsmitteln zuzulassen (SARS-CoV-2-Arzneimittelversorgungsverordnung), mit dem Ziel, Kontakte zu reduzieren, aber gleichzeitig die Versorgung der PatientInnen zu sichern und Drogenbeschaffung sowie -konsum vorzubeugen [32]. Während der epidemischen Lage dürfen bspw. konsiliarisch tätige ÄrztInnen ohne suchtmedizinische Qualifikation mehr als 10 PatientInnen behandeln, die Ausnahme vom Sichtbezug wurde auf 7 Tage ausgedehnt und Take-Home-Verordnungen können ohne persönlichen ärztlichen Kontakt via Postweg oder Botendienst ausgegeben werden [33]. Die zu diesem Zeitpunkt seit zweieinhalb Jahren in Kraft getretenen Neuerungen der 3. BtMVVÄndV stellten eine hilfreiche Grundlage für die COVID-19-bedingten Änderungen der BtMVV dar, die sowohl BehandlerInnen als auch PatientInnen den Umgang mit der Verschreibung und Abgabe von Substitutionsmitteln in diesen Zeiten deutlich erleichtern. Ohne die 3. BtMVVÄndV wären die Ausnahmeregelungen zur Substitution während der Coronapandemie vermutlich nicht möglich gewesen.

\section{Limitationen}

Die Auswahl der Interviewteilnehmenden erfolgte nicht durch eine Zufallsauswahl und ist - wie bei qualitativen Studien überwiegend der Fall - nicht als repräsentativ anzusehen. ÄrztInnen ohne eine suchtmedizinische Weiterbildung, die im Rahmen des Konsiliarverfahrens tätig sind, wurden nicht interviewt, da sie lediglich rund $1 \%$ der SubstitutionspatientInnen behandeln [25]. Lediglich 5 angefragte substituierende ÄrztInnen haben die Interviewanfrage abgelehnt, 2 aus Zeitgründen und 3 aus mangelndem Interesse an einem Interview. Auch wenn die Interviews in ausgewählten KVRegionen mit einer geografisch unterschiedlich guten Versorgung von SubstitutionspatientInnen stattfanden, ist die Studie nicht repräsentativ für das gesamte Bundesgebiet. 


\section{FAZIT FÜR DIE PRAXIS}

Durch die 3. BtMVVÄndV ist die Substitutionsbehandlung Opioidabhängiger für die substituierenden ÄrztInnen rechtlich sicherer und besser an den Behandlungsalltag angepasst worden. Die veränderten Vorschriften sind eine gute Grundlage, um neue ÄrztInnen für eine solche Behandlungsform zu motivieren, reichen alleine jedoch nicht aus. Kooperationen werden zukünftig zunehmend wichtiger. Auch abseits der Metropolen gibt es gute Modelle gelungener Vernetzungen der substituierenden ÄrztInnen untereinander, aber auch mit weiteren Akteuren wie Apotheken, Pflegeeinrichtungen, PSB und staatlichen Institutionen. Wesentlich für die niedergelassenen substituierenden ÄrztInnen ist die Unterstützung durch Substitutionsambulanzen oder Kliniken bei PatientInnen, die in den Praxen nicht gut versorgt werden können. Ein kollegialer Austausch von somatischen und psychiatrischen FachärztInnen ist weiterhin schwierig. Die Behandlung von Abhängigkeitserkrankungen muss einen größeren Raum in der Ausbildung von MedizinerInnen einnehmen, damit deutlich wird, dass eine Substitutionsbehandlung von OpioidpatientInnen weit mehr ist als eine reine medikamentöse ,Ersatztherapie "und einen ganzheitlichen und zugleich individualisierten Behandlungsansatz erfordert.

\section{Finanzielle Förderung}

Die vorliegende Arbeit ist Teil einer vom Bundesministerium für Gesundheit (BMG) finanziell geförderten Studie (Förderkennzeichen ZMVI1-2519DSM206).

\section{Autorinnen/Autoren}

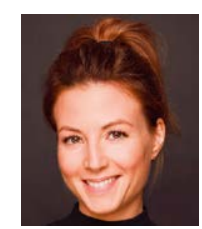

\section{Kirsten Lehmann}

studierte Public Health an der Universität Bremen und ist seit 2014 als wissenschaftliche Mitarbeiterin im Zentrum für Interdisziplinäre Suchtforschung (ZIS) der Universität Hamburg tätig. Neben Medizinischem Cannabis ist ihr Forschungsschwerpunkt die opioidgestützte Substitutionstherapie.

Interessenkonflikt

KL erhielt eine Reisekostenerstattung sowie eine Kostenübernahme von Teilnahmegebühren und Übernachtungskosten von pcm scientific sowie die Kostenübernahme von Teilnahmegebühren von CAMURUS $\mathrm{GmbH}$. SK hat keine Interessenkonflikte. BS hat von CAMURUS GmbH Fördermittel sowie Reisekosten erhalten. UV erhielt innerhalb der letzten 3 Jahre Vortrags-, Beratungshonorar und Reisekostenerstattungen von Mundipharma $\mathrm{GmbH}$ sowie finanzielle Projektförderung und Reisekostenerstattungen von CAMURUS GmbH.
Literatur

[1] Kraus L, Seitz N, Schulte B et al. Schätzung der Anzahl von Personen mit einer Opioidabhängigkeit. Deusches Ärzteblatt Int 2019; 116: 137-143. doi:10.3238/arztebl.2019.0137

[2] Degenhardt L, Bucello C, Mathers B et al. Mortality among regular or dependent users of heroin and other opioids: a systematic review and meta-analysis of cohort studies. Addiction 2010; 106: 32-51

[3] Mattick R, Breen C, Kimber J et al. Methadone maintenance therapy versus no opioid replacement therapy for opioid dependence. Cochrane Database of Systematic Reviews 2009; 3. doi:10.1002/14651858.CD002209.pub2

[4] Busch M, Haas S, Weigl M et al. Langzeitsubstitutionsbehandlung Opioidabhängiger. In: (DIMDI) DIfMDul ed, Schriftenreihe Health Technology Assessment (HTA) in der Bundesrepublik Deutschland. Köln; 2007

[5] Soyka M, Träder A, Klotsche J et al. Mortalität in der langfristigen Substitution: Häufigkeiten, Ursachen und Prädiktoren. Suchtmedizin in Forschung und Praxis 2011; 13: 247-252

[6] Wittchen H, Bühringer G, Rehm J et al. Der Verlauf und Ausgang von Substitutionspatienten unter den aktuellen Bedingungen der deutschen Substitutionsversorgung nach 6 Jahren. Suchtmedizin in Forschung und Praxis 2011; 13: 232-246

[7] Amato L, Minozzi S, Davoli M et al. Psychosocial combined with agonist maintenance treatments versus agonist maintenance treatments alone for treatment of opioid dependence. Cochrane Database of Systematic Reviews 2011; 10. doi:10.1002/14651858. CD004147.pub4

[8] Michels I, Stöver H, Gerlach R. Substitution treatment for opioid addicts in Germany. Harm reduction journal 2007; 4 : 5. doi:10.1186/1477-7517-4-5

[9] Ullmann R. Schaden substituierende Ärzte der Gesellschaft? Suchttherapie 2006; 7: 173-178. doi:10.1055/s-2006-927225

[10] Ullmann R. Strafrechtlicher Umgang mit substituierenden Ärzten aus suchtmedizinischer Sicht. Akzeptanzorientierte Drogenarbeit/ Acceptance-Oriented Drug Work 2010; 7: 1-12

[11] Sachverständigenrat zur Begutachtung der Entwicklung im Gesundheitswesens. Bedarfsgerechte Versorgung - Perspektiven für ländliche Regionen und ausgewählte Leistungsbereiche. In: 2014; https://www. svr-gesundheit.de/index.php?id = 465

[12] Robert Koch-Institut Hrsg. Gesundheitsberichterstattung des Bundes gemeinsam getragen von RKI und Destatis. Gesundheit in Deutschland. In: Berlin: RKI; 2015

[13] Landtag von Baden-Württemberg. Drucksache 15/5712, 12. 09. 2014. Landtag von Baden-Württemberg. 15. Wahlperiode. Antrag der Abg. Dr. Marianne Engeser u. a.. CDU und Stellungnahme des Ministeriums für Arbeit und Sozialordnung, Familie, Frauen und Senioren. Substitution in Baden-Württemberg. In; 2014

[14] Zippel-Schultz B, Specka M, Stöver H et al. Ergebnisse der langjährigen Substitutionsbehandlung Opiatabhängiger - die SubsCare-Studie. Suchttherapie 2019; 20: 72-80

[15] Strada L, Schmidt C, Rosenkranz M et al. Factors associated with health-related quality of life in a large national sample of patients receiving opioid substitution treatment in Germany: A cross-sectional study. Substance abuse treatment, prevention, and policy 2019; 14: 2. doi:10.1186/s13011-018-0187-9

[16] Bundesministerium für Gesundheit. 3. Verordnung zur Änderung der BtMVV. Die Verordnung im Bundesgesetzblatt (nicht druckfähig). Bundesanzeiger 2017: https://www.bgbl.de/xaver/bgbl/start. xav?startbk=Bundesanzeiger_BGBl\&jumpTo=bgbl117s1275.pdf\#_ bgbl_\%1272F\%1272F*\%1275B\%1240attr_id\%1273D\%1227bgbl1117s1275.pdf\%1227\%1275D__1598600639518 
[17] Bundesärztekammer Richtlinie der Bundesärztekammer zur Durchführung der substitutionsgestützten Behandlung Opioidabhängiger. In: Berlin: Bundesärztekammer; 2017: https://www.bundesaerztekammer.de/fileadmin/user_upload/downloads/pdf-Ordner/RL/ Substitution.pdf

[18] Bundesärztekammer Bericht der Bundesärztekammer zur Feststellung des aktuellen Standes der Erkenntnisse der medizinischen Wissenschaft in einer Richtlinie zur Durchführung der substitutionsgestützten Behandlung pioidabhängiger - Methodik und Ergebnisbewertung. In. Berlin: Bundesärztekammer; 2017: https://www.bundesaerztekammer.de/fileadmin/user_upload/downloads/pdf-Ordner/Sucht/ Methodenbericht_Substitution.pdf

[19] Schulte B, Schmidt C, Kuhnigk O et al. Structural barriers in the context of opiate substitution treatment in Germany - a survey among physicians in primary care. Substance abuse treatment, prevention, and policy 2013; 8

[20] Gesundheitsministerkonferenz (GMK). Beschlüsse der 91. GMK. TOP: 10.18 Evaluation der Neuregelung zur substitutionsgestützten Behandlung opioidabhängiger Menschen 2018. https://www. gmkonline.de $/$ Beschluesse. $\cdot$ html id $=727 \&$ jahr $=2018$

[21] Stöver $H$. Barriers to opioid substitution treatment access, entry and retention: a survey of opioid users, patients in treatment, and treating and non-treating physicians. European addiction research 2011; 17: 44-54. doi:10.1159/000320576

[22] Mars S, Bourgois P, Karandinosc G et al. "Every 'Never' I Ever Said Came True": Transitions from opioid pills to heroin injecting. Int J Drug Policy 2014; 25: 257-266. doi:10.1016/j.drugpo.2013.10.004

[23] van Zee A. The promotion and marketing of oxycontin: commercial triumph, public health tragedy. Am J Public Health 2009; 99: 221-227

[24] Stoicea N, Costa A, Periel L et al. Current perspectives on the opioid crisis in the US healthcare system: A comprehensive literature review. Medicine. 2019; 98 20: doi:http://dx.doi.org/10.1097/ MD.0000000000015425

[25] Bundesinstitut für Arzneimittel und Medizinprodukte (BfArM). Bericht zum Substitutionsregister: Januar 2020. 2020. https://www.bfarm.de/ SharedDocs/Downloads/DE/Bundesopiumstelle/SubstitReg/ Subst_Bericht2020.pdf
[26] McNeely J, Drucker E, Hartel D et al. Office-based methadone prescribing: Acceptance by inner-city practitioners in New York. J Urban Health 2000; 77: 96-102. doi:10.1007/BF02350965

[27] Reiß M, Schulte B, Schmidt C et al. Selbst- und Fremdbeurteilung von Depressivität bei opioidabhängigen Patienten in Substitutionsbehandlung Self-Assessment. Suchttherapie 2020; 21: 25-31

[28] Wittchen H, Bühringer G, Rehm J et al. Die soziale, klinische und therapeutische Situation von Substitutionspatienten: Der Status der PREMOS-Patienten bei Studienbeginn (Baseline t1). Suchtmedizin in Forschung und Praxis 2011; 13: 227-231

[29] Lieb M, Wittchen H, Palm U et al. Psychiatric comorbidity in substitution treatment of opioid-dependent patients in primary care: Prevalence and impact on clinical features. Heroin Addict Relat Clin Probl 2010; 12: 5-16

[30] Funke W, Kuhlmann T, Backmund M et al. Substitutionsbehandlung opioidabhängiger Menschen in der medizinischen Rehabilitation: Positionspapier der Deutschen Suchtgesellschaft. Suchttherapie 2020; 21: $39-42$

[31] Jeschke P, Meyer-Thompson H. Informationen zur Opioid-Substitution und Sars-CoV-2/COVID-19. Hinweise für substituierende Ärztinnen und Ärzte. Konferenz der Vorsitzenden von Qualitätssicherungskommissionen der Kassenärztlichen Vereinigungen in Deutschland. In: 2020; https://www.kvno.de/downloads/quali/substitution_corona.pdf

[32] Bundesministerium für Gesundheit. Verordnung über Abweichungen von den Vorschriften des Fünften Buches Sozialgesetzbuch, des Apothekengesetzes, der Apothekenbetriebsordnung, der Arzneimittelpreisverord-nung, des Betäubungsmittelgesetzes und der Betäubungsmittel-Verschreibungsverordnung infolge der SARS-CoV-2-Epidemie (SARS-CoV-2-Arzneimittelversorgungsverordnung). 2020 https://www. bundesgesundheitsministerium.de/fileadmin/Dateien/3_Downloads/ Gesetze_und_Verordnungen/GuV/A/SARS-CoV-2-AMVersorgVO-Entwurf_ mit_Begruendung.pdf

[33] von Blanc A, Meyer-Thompson H, Ullmann R. Kurzfassung der BtMVV-Änderungen während der epidemischen Lage von nationaler Tragweite laut SARS-CoV-2-Arzneimittelversorgungsverordnung vom 20. April 2020; 2020: https://nls-online.de/home16/images/ SARS-CoV-12-Arzneimittelversorgungsverordnung_-_Kurzfassung_ der__BtMVV-Abschnitte_-14-.pdf 